UDC 517.9

\title{
GENERATION OF GRID MULTISCROLL CHAOTIC ATTRACTORS USING CURRENT CONVEYOR
}

\section{ПОРОДЖЕННЯ СІТКИ БАГАТОСПІРАЛЬНИХ ХАОТИЧНИХ АТРАКТОРІВ ЗА ДОПОМОГОЮ КОНВЕЕРА СТРУМУ}

\author{
Y. Lin, C. H. Wang, H. Xu \\ College Inform. Sci. and Eng., Hunan Univ. \\ Changsha 410082, China
}

We study the chaos in a system of three differential equations with piecewise linear right-hand sides, and numerically find chaotic attractors in the system, together with experimentally corresponding modes in an electrical circuit.

Вивчено хаос у системі трьох диференціальних рівнянь з кусково-лінійною правою частиною та чисельно знайдено хаотичні атрактори системи разом з експериментально відповідними режимами електричної схеми.

1. Introduction. Over the last four decades, chaos has been intensively studied within the science, mathematics, engineering communities and so on [1]. Recently, the design and circuit implementation of chaotic oscillators, in particular, generating various complex multiscroll chaotic attractors by using some simple electronic devices, have been of an increasing interest due to their applications in various chaos-based technologies. Many methods to generate multiscroll chaotic attractors have been proposed, some by introducing additional breakpoints in the piecewise-linear characteristic of the nonlinear resistor of Chua's circuit [2-5], some by replacing the original nonlinear resistor by other nonlinear functions, such as: cubic function [6], sinusoidal function [7], and some by other kinds of circuits, such as Jerk circuit [8], hysteresis series method [10], staircase function series and saturated nonlinear function series (SNFS) approach [12,18]. These approaches have been experimentally verified, but their functions were mainly built with Op amps, Operational Trans-conductance Amplifiers (OTAs), or specific integrated circuits $[11,13,16]$. However, it is well known that the dynamic range with voltage mode circuit is limited, it is almost impossible to generate chaotic attractors with a large number of scrolls $[8,14]$. Moreover, the op amp's nonideal phase characteristics limit its operating frequency. Aiming at high-frequency and a large number of scrolls chaotic signals, some methods have been proposed to generate chaotic attractors employing Current Feedback Operational Amplifiers (CFOAs) or CCIIs which can provide high speed and have large dynamic range $[17,19]$. But they used CFOAs and voltage comparators to implement function in [17]. In [19] they used only CCII as active device, but they only realized 1-D chaotic attractors. It is very interesting to ask whether or not multidirectional grid-scroll chaotic attractors employing only current conveyor at high frequency can be realized? This paper gives a positive answer to this question.

The rest of the paper is organized as follows. In Section 2, we discuss 2-D $(n \times m)$-grid scroll attractors obtained by applying staircase nonlinear functions. Recursive formulas to compute chaotic attractor parameters are deduced also. In Section 3, the nonlinear dynamical system is

(C) Y. Lin, C. H. Wang, H. Xu, 2013 
synthesized employing CCIIs, EWB simulation results are accomplished. In Section 4, experimental tests in both time and frequency domains are given. Conclusions are drawn finally in Section 5.

2. 2-D grid scroll chaotic attractors. Consider the following three-order nonlinear autonomo-

us system:

$$
\begin{aligned}
& \dot{x}=y-f(y), \\
& \dot{y}=z, \\
& \dot{z}=-a x-b y-c z+d f(x) .
\end{aligned}
$$

Where $x, y$ and $z$ are state variables, $a, b, c$ and $d$ are positive real constants, $f(x)$ is modeled as staircase nonlinear function series given by

$$
f(x)=A_{x}\left[\sum_{i=0}^{\left(N_{x}-3\right) / 2}\left(\operatorname{sgn}\left(x+(2 i+1) A_{x}\right)+\operatorname{sgn}\left(x-(2 i+1) A_{x}\right)\right]\right.
$$

for one odd number of scrolls $\left(N_{x} \geq 3\right)$, or

$$
f(x)=A_{x}\left[-\operatorname{sgn}(x)+\sum_{i=0}^{\left(N_{x}-2\right) / 2}\left(\operatorname{sgn}\left(x+2 i A_{x}\right)+\operatorname{sgn}\left(x-2 i A_{x}\right)\right)\right]
$$

for one even number of scrolls $\left(N_{x} \geq 2\right)$, where $A_{x}>0$ and

$$
\operatorname{sgn}(x)= \begin{cases}1, & \text { if } \quad x>0 \\ 0, & \text { if } \quad x=0 \\ -1, & \text { if } \quad x<0\end{cases}
$$

The function $f(y)$ can also be modeled with (2) or (3), but by substituting the $x$-indexes by $y$-indexes. The system (1) has the potential to create 2-D $N_{x} \times N_{y}$-scroll chaotic attractors for some suitable parameters $a, b, c$ and $d$, here $a=b=c=d=0.7$. Staircase waveforms for (2) and (3) are shown in Fig. 1, we can see that chaotic attractor parameters, including the width and equilibrium points, can be estimated beforehand by using nonlinear system parameters along with real physical active device parameters.

Here, $D R_{x}, W_{x}, N_{x}$ are the dynamic range, Width and $N$ scrolls in $x$-axes respectively, and $D R_{y}, W_{y}, N_{y}$, are in $y$-axes. By considering $D R_{y}=D R_{x} / N_{x}$, the width of each scroll is estimated as $W_{x}=D R_{x} /\left(N_{x} N_{y}\right)$ (except for the outside edge scroll) and the periods of the staircase nonlinear functions are given by $W_{x}=2 A_{x}$, then $A_{x}=D R_{x} / 2\left(N_{x} N_{y}\right)$, similarly $W_{y}=D R_{y} / N_{y}$, then $A_{y}=D R_{y} / 2 N_{y}$. More, one can rigorously deduce a set of recursive formulas to equilibrium points $\left(E_{j}\right)$ and breakpoints $\left(B_{j}\right)$ for both $x$-axes and $y$-axes as follows:

(1) $X$-axes, $N_{x}=$ odd and $N_{y}=$ odd, $N_{x}, N_{y}>1$,

$$
\pm E_{j}= \pm \sum_{j=1}^{\frac{N_{x} N_{y}-1}{2}}\left(E_{j-1}+2 A_{x}\right), \quad E_{0}=0
$$




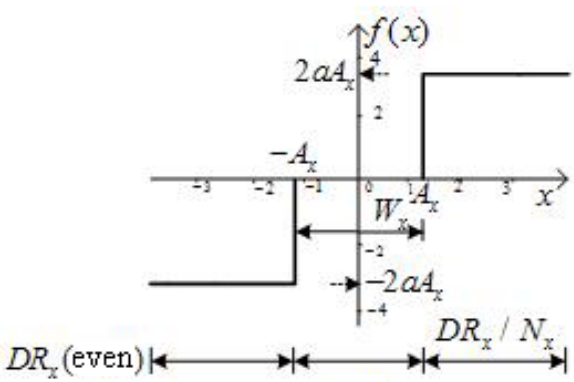

(a)

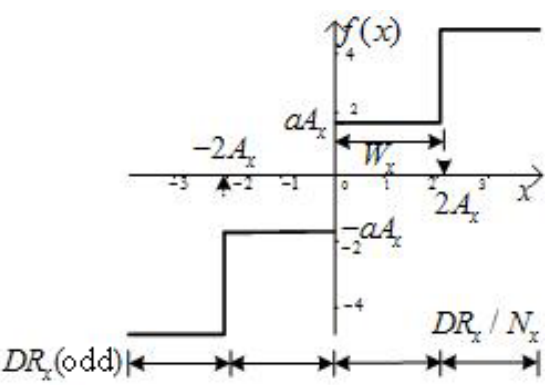

(b)

Fig. 1. (a) Staircase functions for: $N=3$ by using (2). (b) Staircase functions for: $N=4$ by using (3).

For other combinations,

$$
\pm E_{j}= \pm \sum_{j=1}^{\frac{N_{x} N_{y}}{2}}\left(E_{j-1}+2 A_{x}\right), \quad E_{0}=-A_{x}
$$

The breakpoints are computed by

$$
\pm B_{j+1}= \pm \sum_{j=0}^{\frac{N_{x}-3}{2}}(2 j+1) A_{x}
$$

for odd chaotic attractors, $N_{x} \geq 3$, and

$$
\pm B_{j+1}= \pm \sum_{j=0}^{\frac{N_{x}-2}{2}} 2 j A_{x}
$$

for even chaotic attractors, $N_{x} \geq 2$.

(2) $Y$-axes

$$
\begin{gathered}
\pm E_{j+1}= \pm \sum_{j=0}^{\frac{N_{y}-3}{2}}\left(E_{j}+2 A_{y}\right), \quad E_{0}=0, \quad N_{y} \quad \text { is odd } \geq 3, \\
\pm E_{j+1}= \pm \sum_{j=0}^{\frac{N_{y}-2}{2}}\left(E_{j}+2 A_{y}\right), \quad E_{0}=-A_{y}, \quad N_{y} \quad \text { is even } \geq 2,
\end{gathered}
$$




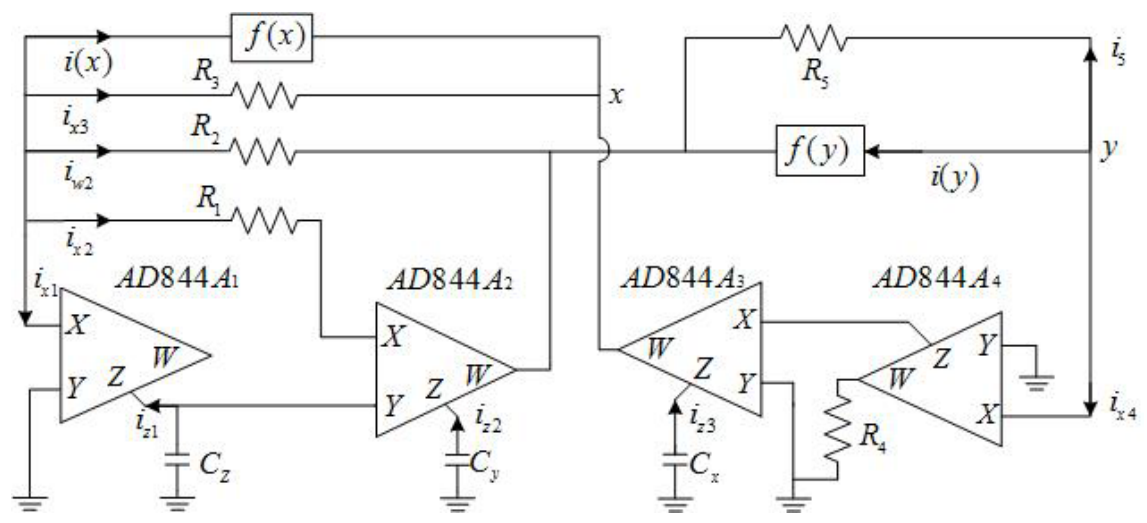

Fig. 2. Block circuit diagram of 2-D grid-scroll chaotic circuits with CCIIs.

and the breakpoints are computed by

$$
\begin{gathered}
\pm B_{j+1}= \pm \sum_{j=0}^{\frac{N_{y}-3}{2}}(2 j+1) A_{y}, \quad N_{y} \quad \text { is odd } \geq 3 \\
\pm B_{j+1}= \pm \sum_{j=0}^{\frac{N_{y}-2}{2}} 2 j A_{y}, \quad N_{y} \quad \text { is even } \geq 2 .
\end{gathered}
$$

Because (4)-(11) are all dependent on the DRs and the number of scroll in the $x$-axes and $y$-axes respectively, it is not necessary to carry out a scaling in (2) and (3) [15]. By adjusting these parameters, one can arbitrarily design the width and the equilibrium points, to generate various grid scroll attractors.

3. Circuit implementation for 2-D $(n \times m)$-grid scroll chaotic attractors. The second-generation current conveyor (CCII) has been considered as a versatile block designed for currentmode signal processing, and widely used to design linear and nonlinear circuits $[15,19]$. The CCII+ is commercially available as CFOA such as AD844, which includes a CCII+ in cascade connection with a voltage buffer in its internal structure, is recognized for its excellent performance in high-speed and high slew-rate. For this case, the system (1) is synthesized with AD844 in configuration of CCIIs, as shown in Fig. 2.

The dynamic equations of the designed grid-scroll circuits are rigorously derived from the circuit diagram Fig. 2. Here, AD844A3 cascaded AD844A4 forms a CCII-. Using the node analysis approach from circuit theory, by considering parasitic elements associated with the main input-output terminals of AD844, whose typical values are $R_{y j}=10 M \Omega, R_{x j}=50 \Omega$, $R_{z j}=3 M \Omega, R_{w j}<15 \Omega C_{y j}=2 p F, C_{z j}=4.5 p F$. Here, for simplification, the parasitic resistances $R_{W 1}, R_{W 2}$ and $R_{W 3}$ are not considered. On account of $i_{z 1}=i_{x 1}, i_{z 2}=i_{x 2}, i_{z 3}=$ 


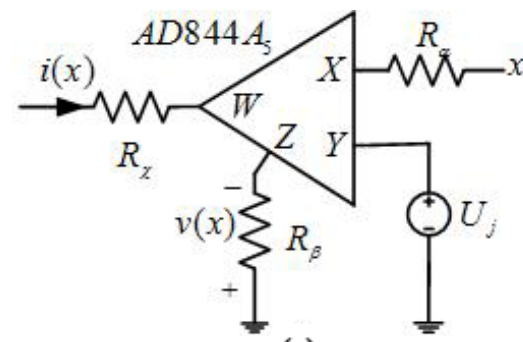

(a)

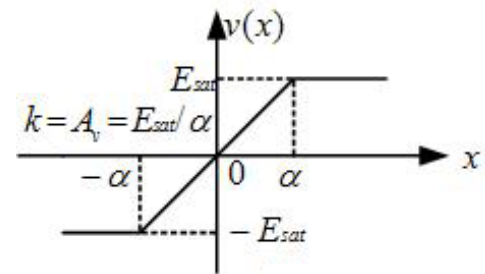

(c)

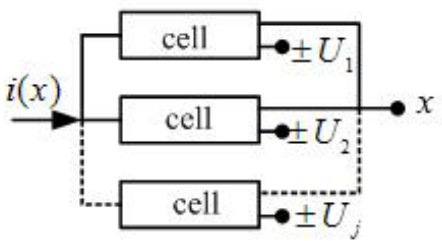

(b)

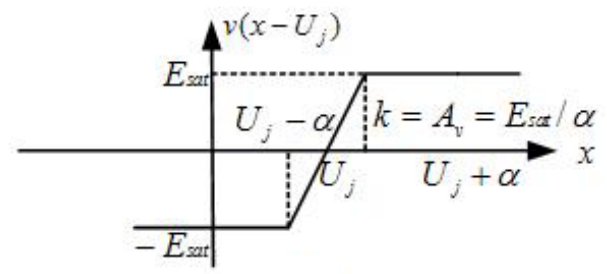

(d)

Fig. 3. (a) Basic cell to design SNFS. (b) General structure to generate (2) and (3).

(c) Voltage saturated function $v(x)$. (d) Shifted voltage saturated function $v(x-U j)$.

$=-i_{x 4}$, we can obtain the nonlinear system as follows:

$$
\begin{aligned}
\dot{x}= & \frac{y}{R_{5}\left(C_{x}+C_{z 3}\right)}-\frac{i(y)}{C_{x}+C_{z 3}}, \\
\dot{y}= & \frac{z}{\left(R_{1}+R_{x 2}\right)\left(C_{y}+C_{z 2}\right)}, \\
\dot{z}= & -\frac{x}{R_{3}\left(C_{z}+C_{z 1}+C_{y 2}\right)}-\frac{y}{R_{2}\left(C_{z}+C_{z 1}+C_{y 2}\right)}-\frac{z}{\left(R_{1}+R_{x 2}\right)\left(C_{z}+C_{z 1}+C_{y 2}\right)}+ \\
& +\frac{i(x)}{C_{z}+C_{z 1}+C_{y 2}} .
\end{aligned}
$$

By comparing (1) with (12), the following relations are obtained:

$$
\begin{aligned}
& a\left(C_{z}+C_{z 1}+C_{y 2}\right)=C_{x}+C_{z 3}=C_{y}+C_{z 2}, \\
& R_{5}=\left(R_{1}+R_{x 2}\right)=R_{3}=R_{2}=\frac{1}{a\left(C_{z}+C_{z 1}+C_{y 2}\right)}, \\
& f(x)=R_{3} i(x), \\
& f(y)=R_{5} i(y) .
\end{aligned}
$$

It's known that the staircase nonlinear function series and given by (2) and (3) can not be designed with real active devices, but they can be approached with SNFS by considering that the slope is infinity and the switching points are zero [12]. The SNFS can be designed with CCII $+s$ configured with a high-gain voltage amplifier [19], as shown in Fig. 3(a). If parasitic elements are considered in Fig. 3(a), in the linear region the shifted voltage saturated function is given by: 


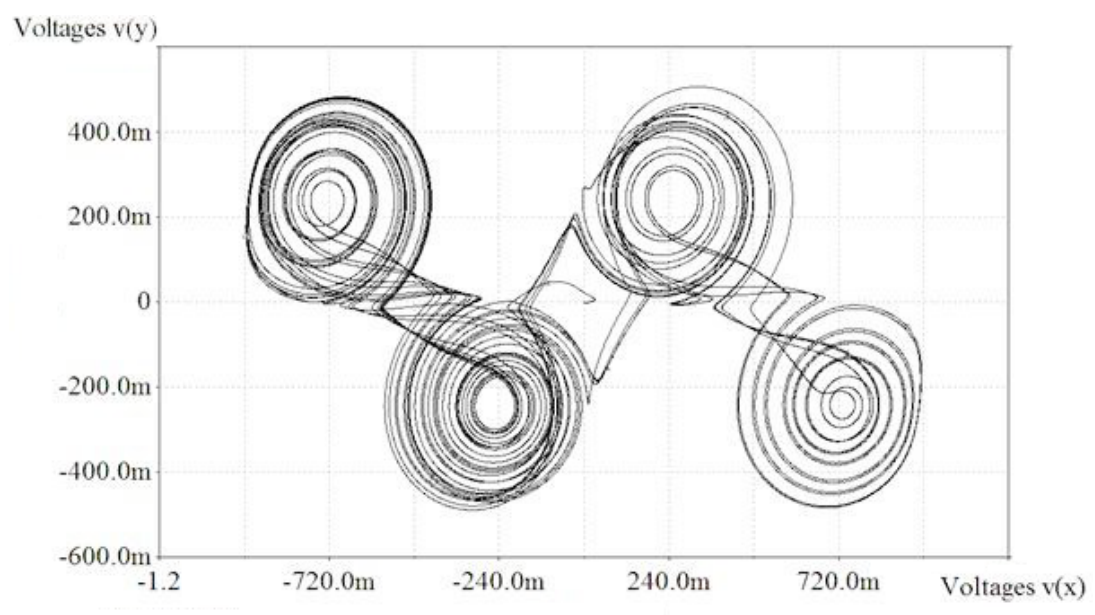

Fig. 4. $(2 \times 2)$-grid scrolls EWB simulation results.

$$
v\left(x-U_{j}\right)=\frac{R_{\beta} / / R_{z 5}}{R_{\alpha}+R_{x 5}}\left(x-U_{j}\right)=A_{v}\left(x-U_{j}\right) .
$$

Figure 3(c) shows the voltage saturated function $v(x)$ and Fig. 3(d) shows the shifted voltage saturation function $v\left(x-U_{j}\right)$, where $k$ is the slope, and $\pm \alpha$ are the switching points. We have fixed $R_{\alpha}=1 K \Omega$ and $R_{\beta}=1 M \Omega$ to minimize the effect of $R_{x 5}$ and $R_{z 5}$ then $A_{v} \approx 57.1 d B$. When the supply voltages of the positive and negative electrical sources are $\pm E= \pm 4.5 \mathrm{~V}$, the experiment results show $E_{\text {sat }} \approx 1.05 \mathrm{~V}, \alpha \approx 4.9 \mathrm{mV}$. When $\pm E= \pm 5 \mathrm{~V}$, then $E_{\mathrm{sat}} \approx 1.45 \mathrm{~V}$, $\alpha \approx 5.6 \mathrm{mV}$. The current saturated behavior is determined by the saturation voltage of AD844 and $R \chi$, such that $i(x) \approx v(x) / R_{\chi}$. One can construct more complex saturated function series circuit by using the basic cell shown in Fig. 3(a) with different comparing voltages $U_{j}\left( \pm U_{j}=\right.$ $\left.= \pm B_{j}(x, y)\right)$ connected in parallel shown in Fig. 3(b). In Fig. 2, two voltage followers are used to separate $R_{3}$ and $i(x)$ from $x$-state variable, $R_{2}$ from $z$-state variable. In order to transform the saturation voltage to saturation current, one voltage follower is used between the $Z$ and $W$ terminals from Fig. 3(a).

3.1. $(2 \times 2)$-grid scroll chaos attractors generation. To generate $(2 \times 2)$-grid scroll attractors, one basic cell should be used to design $i(x)$ and one basic cell should be used to design $i(y)$. The dynamic range mainly depends on the bias power supplies. Because AD844 can be operated from $4.5 \mathrm{~V}$ to $18 \mathrm{~V}$ voltage supplies, the experiment results show that the dynamic ranges are approximately from $2 \mathrm{~V}$ to $30 \mathrm{~V}$. We can set the bias supply voltages to $4.5 \mathrm{~V}$, the experiment results show that the DR of AD844 is about $2 V$. So we assumed the following input variables: $D R x=2 V, N x=2, N y=2, a=0.7$. Attractor parameters are calculated by using (3), (5), (7), (9) and (11) and are given in Table 1. Afterwards, by using (13) and by considering parasitic elements, the numerical values of the passive elements from Fig. 2 are approximated and given in Table 2. To minimize the effects of the parasitic resistors, the values of should be large. Making use of the excellent performance of the CCII at high frequencies, we can scale down the capacitors $C_{x}, C_{y}, C_{z}$ to extend the operating frequency by a factor $q=C_{x, y, z}^{\text {old }} / C_{x, y, z}^{\text {new }}$ with the condition of $C_{x, y, z}^{\text {new }}>10\left(C_{z 1, z 2, z 3}+C_{y 2}\right)$ [15], EWB simulation result in the state space is shown in Fig. 4 for $C_{x}=C_{y}=C_{z}=143 p F$. In order to exploit the capabilities offered by CCIIs and to improve the frequency response, CMOS technology should be used [19]. 


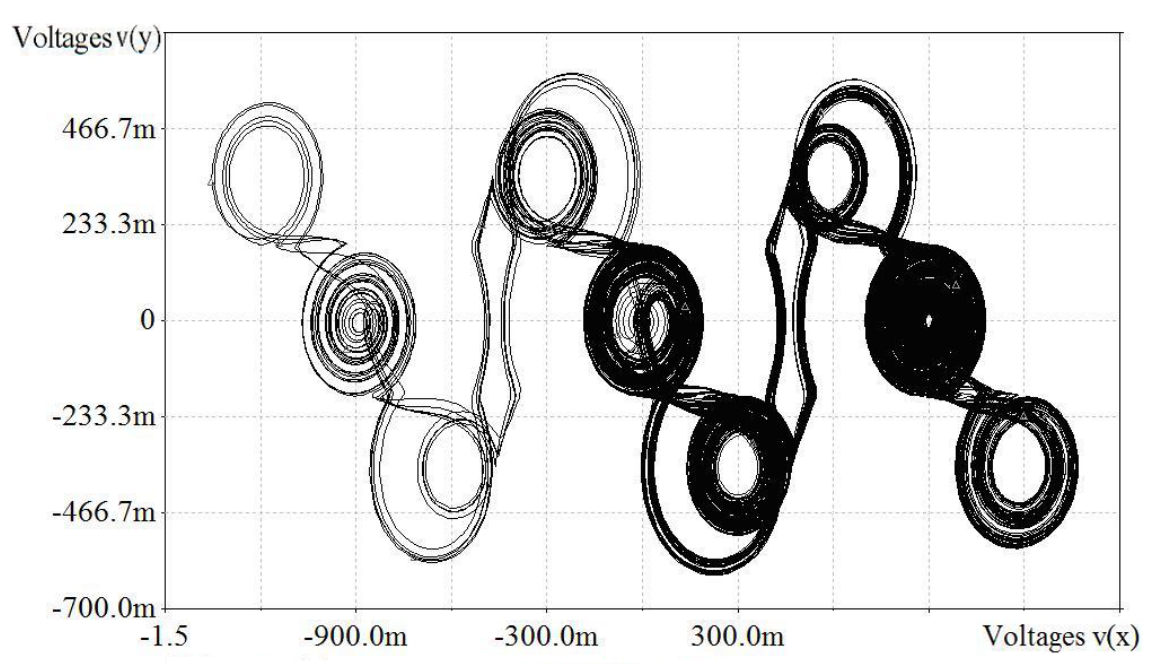

Fig. 5. $(3 \times 3)$-grid scrolls EWB simulation results.

Table 1. $(2 \times 2)$-grid scroll and $(3 \times 3)$-grid scroll attractor parameters

\begin{tabular}{c|c|c|c}
\hline$(2 \times 2)$-grid scroll & Bias $\pm 4.5 \mathrm{~V}$ & $(3 \times 3)$-grid scroll & Bias $\pm 5 \mathrm{~V}$ \\
\hline $\begin{array}{c}W_{x}=W_{y}=0.5 \mathrm{~V} \\
\pm U_{j}= \pm B_{j}(x, y)\end{array}$ & $\pm E_{j}(x, y)$ & $\begin{array}{c}W_{x}=W_{y}=0.31 \mathrm{~V} \\
\pm U_{j}= \pm B_{j}(x, y)\end{array}$ & $\pm E_{j}(x, y)$ \\
\hline \multirow{2}{*}{0,0} & $0.25,0.25$ & $\pm 0.45, \pm 0.15$ & 0,0 \\
& $0.75,-0.25$ & & $0.31,-0.31$ \\
& $-0.75,0.25$ & & $0.62,0.31$ \\
& $-0.25,-0.25$ & & $\pm 0.93,0$ \\
& & & $-1.21,0.31$ \\
& & & $-0.62,-0.31$ \\
& & & $-0.31,0.31$ \\
\hline
\end{tabular}

3.2. $(3 \times 3)$-grid scroll chaos attractors generation. To generate $(3 \times 3)$-grid scroll attractors, two basic cells connected in parallel should be used to design $i(x)$ and two basic cells connected in parallel should be used to design $i(y)$. In the experiment, we set the bias supply voltages $\pm 5 \mathrm{~V}$ to generate $(3 \times 3)$-grid scroll attractors, and the experiment results show that the DR of AD844 is about 2.8V. So we assumed the following input variables: $D R=2.8 \mathrm{~V}, N x=3$, $N y=3, a=0.7$. Attractor parameters are calculated by using (2), (4), (6), (8) and (10), and are given in Table 1. The numerical values of the passive elements introduced in Table 2. EWB simulation result in the state space is shown in Fig. 5.

4. Experimental results. With the same numerical values used in simulation, we experimentally test the proposed circuit shown in Fig. 2 by using discrete components, for convenient adjustment and higher precision, all resistors are precisely adjustable resistors or potentiometers. 
Table 2. Numerical values of passive elements for Fig. 2

\begin{tabular}{c|c|c}
\hline Passive elements & $(2 \times 2)$-grid scroll & $(3 \times 3)$-grid scroll \\
\hline$C_{x}=100 u F$ & $E_{\text {sat }} \approx 1.05 \mathrm{~V}$ & $E_{\text {sat }} \approx 1.45 \mathrm{~V}$ \\
$C_{y}=100 u F$ & $R_{\chi x}=22.5 \mathrm{~K} \Omega$ & $R_{\chi x}=32 \mathrm{~K} \Omega$ \\
$C_{z}=143 u F$ & $R_{\chi y}=45 K \Omega$ & $R_{\chi y}=96 \mathrm{~K} \Omega$ \\
$R_{1}=10 \mathrm{~K} \Omega$ & & \\
$R_{2}=10 \mathrm{~K} \Omega$ & & \\
$R_{3}=10 \mathrm{~K} \Omega$ & & \\
$R_{5}=10 \mathrm{~K} \Omega$ & & \\
\hline
\end{tabular}

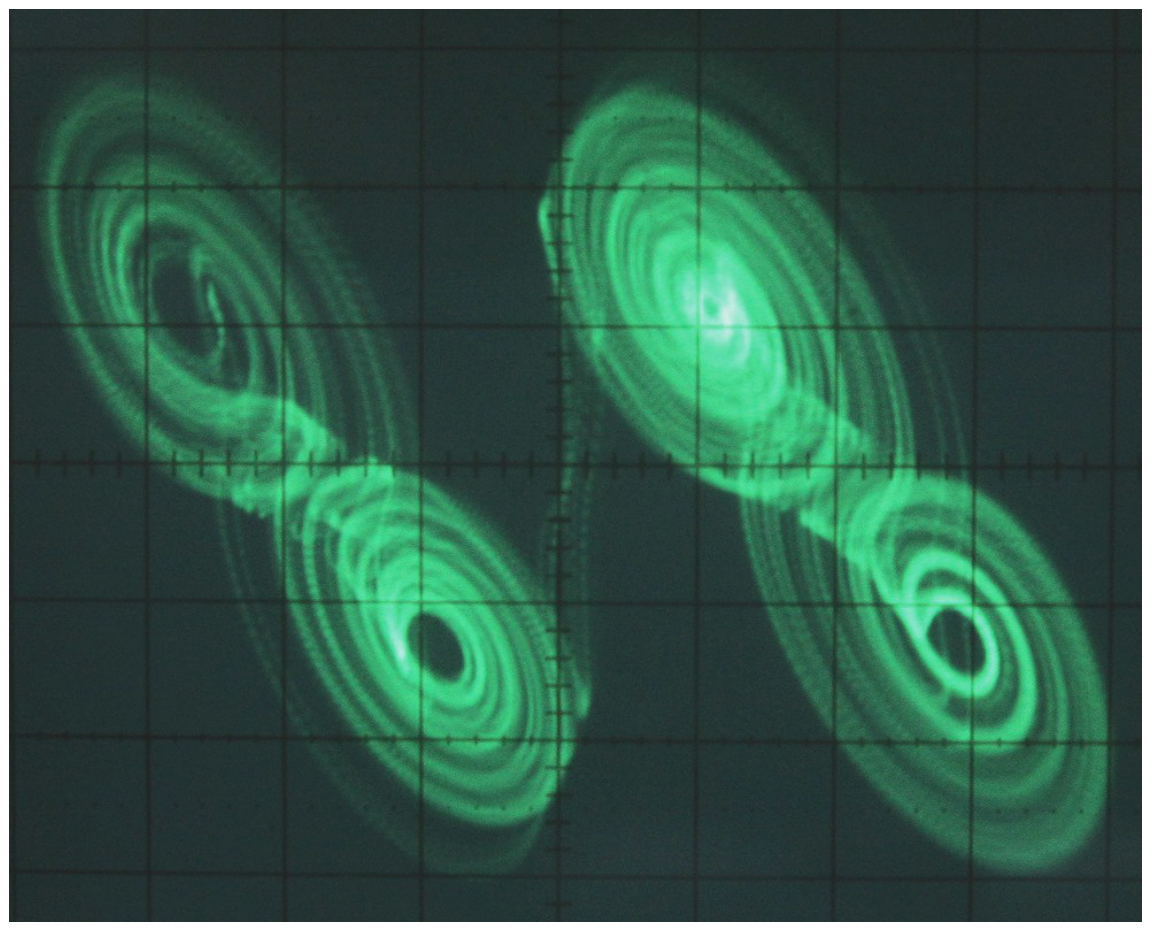

Fig. 6. Experimental verification of $(2 \times 2)$-grid scroll attractors; horizontal-axes: $0.2 \mathrm{~V} / \mathrm{div}$ and vertical-axes: $0.2 \mathrm{~V} / \mathrm{div}$. 


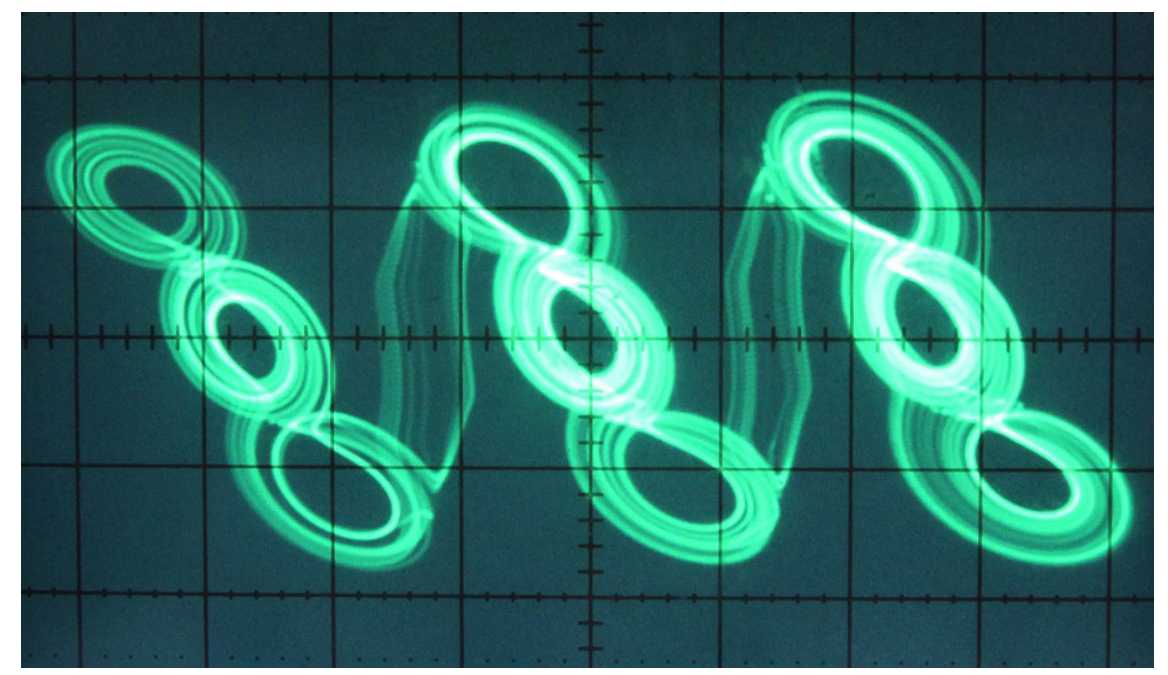

Fig. 7. Experimental verification of $(3 \times 3)$-grid scroll attractors; horizontal-axes: $0.3 \mathrm{~V} / \mathrm{div}$ and vertical-axes: $0.2 \mathrm{~V} / \mathrm{div}$.

Table 3. Measured attractors parameters

\begin{tabular}{|c|c|c|c|}
\hline$(2 \times 2)$-grid scroll & Bias $\pm 4.5 \mathrm{~V}$ & $(3 \times 3)$-grid scroll & Bias $\pm 5 V$ \\
\hline $\begin{array}{c}W_{x}=0.4 V, W_{y}=0.55 \mathrm{~V} \\
\pm U_{j}= \pm B_{j}(x, y)\end{array}$ & $\pm E_{j}(x, y)$ & $\begin{array}{c}W_{x}=W_{y}=0.31 V \\
\pm U_{j}= \pm B_{j}(x, y)\end{array}$ & $\pm E_{j}(x, y)$ \\
\hline 0,0 & $\begin{array}{c}0.2,0.25 \\
0.6,-0.25 \\
-0.6,0.25 \\
-0.2,-0.25\end{array}$ & $\pm 0.45, \pm 0.15$ & $\begin{array}{c}0,0 \\
0.28,-0.25 \\
0.6,0.25 \\
\pm 0.75,0 \\
-1.05,0.25 \\
1.05,-0.25 \\
-0.6,-0.25 \\
-0.28,0.25\end{array}$ \\
\hline
\end{tabular}

Fig. 6 and Fig. 7 respectively show the experimental result of $(2 \times 2)$-grid scroll attractors and $(3 \times 3)$-grid scroll attractors corresponding to $x(t)-y(t)$ plane, Table 3 shows the measured attractor parameters. From Tables 1 and 3 we note that errors are obtained when $E_{j}$ are computed and compared with measured data. This is because the SNFS designed with CCII+s configured with a high-gain voltage amplifier are ideally modeled as staircase nonlinear functions. Therefore, this limitation can be solved if the SNFS are modeled as in [13]. There is a trade-off between the number of scroll and the frequency of operation depending on the behavior in frequency of the cells used to design the SNFS. After several experimental tests, 


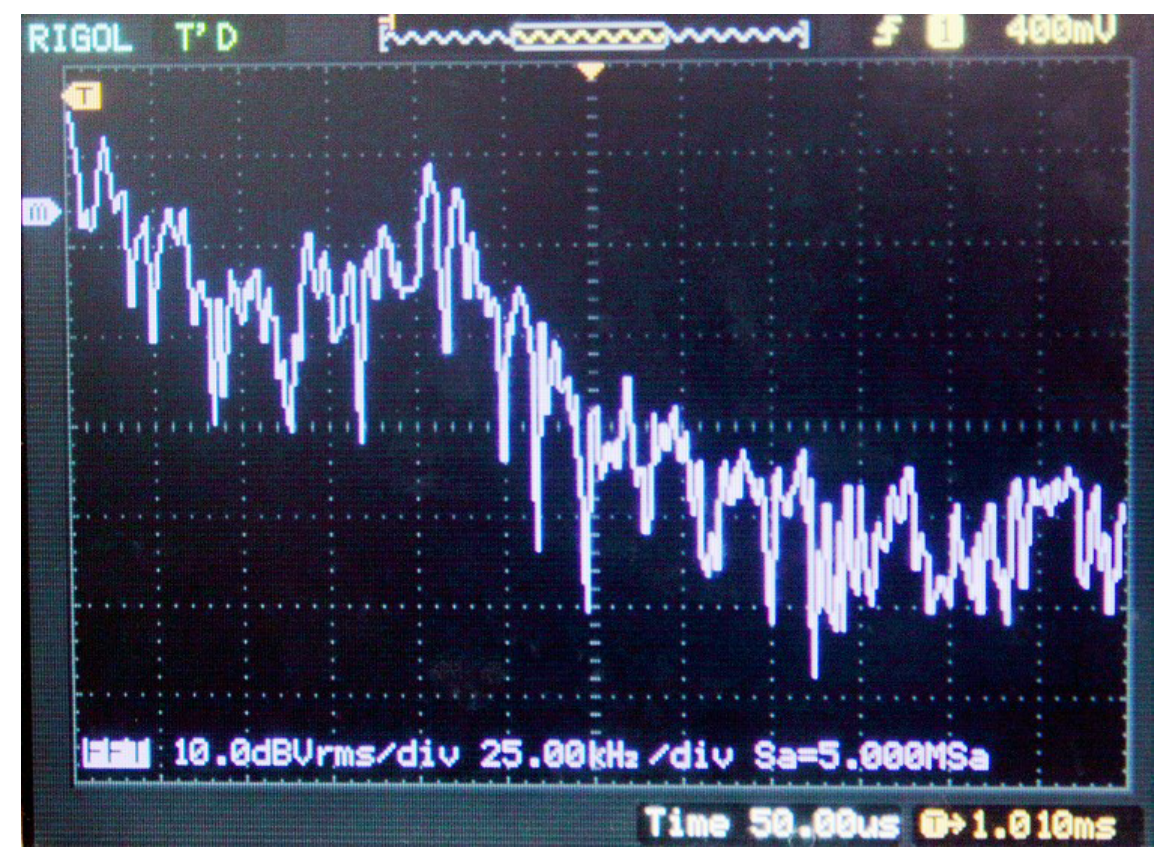

Fig. 8. Experimental measurement of the chaotic spectrum of the $(2 \times 2)$-grid scrolls.

we observe that the behavior in frequency of the $(3 \times 3)$-grid scroll chaotic attractors is lower than that of the $(2 \times 2)$-grid scroll chaotic attractors on even ground. Here only the $(2 \times 2)$-grid scroll chaotic attractors frequency spectrum is shown in Fig. 8 and it is centered to $105 \mathrm{kHz}$.

5. Conclusions. An approach to achieve grid multiscroll chaotic attractors using CCIIYัAs is proposed. The approach has many advantages over the classical methods: firstly, we may not carry out a scaling of the DR associated with SNFS because $(n \times m)$-grid scrolls parameters are approximated by using nonlinear system parameters along with the DR associated with physical active devices. Secondly, we can deduce recursive formulas to approximate $E_{j}$ and $B_{j}$ depending on the DR and the number of scrollthus, a maximum number of scrolls can be obtained to any given DR. Thirdly, the proposed circuit requires few active and passive elementand can operate at high frequency as well as low bias voltages compared with the method introduced in $[10,13,14,17]$. Experimental results for 2-D $(2 \times 2)$-grid scroll and $(3 \times 3)$-grid scroll attractors have been provided to verify the agreement with numerical simulations.

1. Lu J. H., Yu S. M., Leung H. Experimental verification of multidirectional multiscroll chaotic attractors // IEEE Trans. Circuits and Syst. - 2006. - 53. - P. 149-165.

2. Suykens J. A. K., Huang A., Chua L. O. A family of $n$-scroll attractors from a generalized Chua's circuit // Int. J. Electron. Commun. - 1997. - 51. - P. $131-138$.

3. Yalcin M. E., Ozoguz S., Suykens J. A. K. n-Scroll chaos generators: a simple circuit model // Electron. Lett. 2001. - 37. - P. $147-148$.

4. Chua L. O. The genesis of Chua's circuit // AEU Int. J. Electron. Commun. - 1992. - 46. - P. $250-257$.

5. Tlelo-Cuautle E., Munoz-Pacheco J. M., Martnez Carballido J. Appl. Math. Comput. - 2007. - 194. - P. 612. 
6. Suykens J. A. K., Chua L. O. n-Double scroll hypercubes in 1-D CNNs // Int. J. Bifurc. Chaos. - 1997. - 7. P. $1873-1885$.

7. Tang K. S., Zhong G. Q., Chen G., Man K. F. Generation of $n$-scroll attractors via sine function // IEEE Trans. Circuits and Syst. - 2001. - 48. - P. 1369-1372.

8. Yu S. M., Leung J. L., and H. Design and implementation of n-scroll chaotic attractors from a general jerk circuit // IEEE Trans. Circuits and Syst. - 2005. - 52. - P. 1459-1477.

9. Tanmoy B., Biswajit K., Bishnu C. S. Chaotic electronic oscillator from single amplifier biquad // AEU Int. J. Electron. Commun. - 2012. - 66. - P. 593-597.

10. Nakagawa S., Saito T. An RC OTA hysteresis Chaos generator // IEEE Trans. Circuits and Syst. - 1996. 43. - P. 1019-1021.

11. Elwakil A. S., Kennedy M.P. Construction of classes of circuit independent chaotic oscillators using passiveonly nonlinear devices // IEEE Trans. Circuits and Syst. - 2001. - 48. - P. 289-307.

12. Yu S., Tang W. K. S., Chen G. Generation of nscroll attractors under a Chua-circuit framework // Int. J. Bifurc. Chaos. - 2007. - 17. - P. $14-19$.

13. Chao W. F., Cai L., Zhang L. S. A study on hyperchaotic system implemented by SETMOS // Microelectron. J. - 2011. - 42. - P. 409-414.

14. Smith K. C., Sedra A. The current conveyor-a new circuit building block // IEEE Proc. - 1968. - 56. P. $1368-1369$.

15. Hasan S., Khan I. A. Multi-scroll with current conveyor // IEEE Multimedia. - 2009. - 4. - P. 229-232.

16. Han J.L.F., Yu X., Chen G. Generating 3-D multi-scroll chaotic attractors: A hysteresis series switching method // Automatica. - 2004. - 40. - P. 1677-1687.

17. Yalcin M. E., Suykens J. A. K., Vandewalle J., Ozoguz S. Families of scroll grid attractors // Int. J. Bifurc. Chaos. - 2002. - 12. - P. 19-25.

18. Chen J. L. G., Yu X., Leung H. Design and analysis of multi-scroll chaotic attractors from saturated function series // IEEE Trans. Circuits and Syst. - 2004. - 51. - P. 2476-2490.

19. Sanchez-Lopez C., Trejo-Guerra R., Munoz-Pacheco J.M. N-scroll attractors from saturated function series employing CCII+s // Nonlinear Dynam. - 2010. - 61. - P. 331-341.

Received 11.09.12 